MATHEMATICS OF COMPUTATION

Volume 68, Number 227, Pages 1097-1108

S $0025-5718(99) 01052-2$

Article electronically published on February 13, 1999

\title{
NUMERICAL DETECTION OF SYMMETRY BREAKING BIFURCATION POINTS WITH NONLINEAR DEGENERACIES
}

\author{
KLAUS BÖHMER, WILLY GOVAERTS, AND VLADIMÍR JANOVSKÝ
}

\begin{abstract}
A numerical tool for the detection of degenerated symmetry breaking bifurcation points is presented. The degeneracies are classified and numerically processed on 1-D restrictions of the bifurcation equation. The test functions that characterise each of the equivalence classes are constructed by means of an equivariant numerical version of the Liapunov-Schmidt reduction. The classification supplies limited qualitative information concerning the imperfect bifurcation diagrams of the detected bifurcation points.
\end{abstract}

\section{INTRODUCTION}

We consider equivariant vector fields depending on several parameters. The aim is the numerical computation of symmetry breaking (steady state) bifurcation points with higher codimension. We concentrate on nongeneric behaviour in the leading terms of the Taylor expansion of the bifurcation equation. Let us call this phenomenon the nonlinear degeneracy to distinguish it, say, from the mode interaction that is a typical linear degeneracy. An example of symmetry breaking bifurcation points of this kind is presented in [6, XV, Case Study 5].

The qualitative analysis in [6] is based on contact equivalence of bifurcation problems. It classifies bifurcation problems by their codimension and a normal form. It allows us to understand the bifurcation point as a local organizing center of the vector field via a universal unfolding of the relevant normal form. Though the basic tools are developed, a systematic classification of symmetry breaking bifurcations with nonlinear degeneracies is missing. Certainly, it would be tedious and highly dependent on the particular group of the underlying symmetry. We refer to [4] for recent progress in this direction that exploits Computer Algebra.

Since we need a well-defined object of the computation, we introduce a classification. It is based on a qualitative classification of the secondary branches that break the symmetry of the problem. Essentially, it leads to the classification of

Received by the editor February 16, 1996 and, in revised form August 8, 1997 and December 16, 1997.

1991 Mathematics Subject Classification. Primary 65 H 10, 58 C 27, 47 H 15, 20 C 30.

Key words and phrases. Symmetry-breaking bifurcation, nonlinear degeneracy, bordered matrices, generalised Liapunov-Schmidt reduction, Newton-like method, pathfollowing.

The first author was partially supported by the Volkswagen Foundation and the Deutsche Forschungsgemeinschaft.

The second author was partially supported by the Fund for Scientific Research F.W.O., Gent, Belgium.

The third author was partially supported by the grants GAČR 201/98/0528 and GAUK 96/199. 
$\mathbf{Z}_{2}$-equivariant (scalar) bifurcation problems (see [5]). From this point of view, the 1-D action of the symmetry group on the tangent to the secondary branch is the only relevant group theoretic input.

Obviously, such a classification does not yield a normal form and its unfolding. On the other hand, we obtain a comparatively simple tool to ascend/descend a predefined hierarchy of singular points using standard numerical techniques (namely, pathfollowing). To understand the organizing role of a detected bifurcation point, we combine the qualitative information (concerning the behaviour of the secondary branches) with some additional numerical effort to discover the full bifurcation diagram.

In Section 2, we review basic facts concerning the spontaneous symmetry breaking and clearly formulate the classification idea. In Section 3, we resume the classification results for the equivalence classes up to codim $=3$. We shall essentially follow [5]. For computational purposes, we modify the list of $\mathbf{t}$ and $\mathbf{Z}_{2}$-equivalence classes in the spirit of [7].

Our aim is the numerical detection and computation of symmetry breaking bifurcation points with nonlinear degeneracies. In Section 4 , we briefly recall the equivariant version of the generalised Liapunov-Schmidt reduction (see [8]). The reduction is applied in order to construct the relevant test functions for each item of the classification list.

We conclude with a numerical example in Section 5.

\section{Symmetry BREAKING BIFURCATION SCENARIO}

Let $F: \mathbb{R}^{N} \times \mathbb{R}^{1} \rightarrow \mathbb{R}^{N}$ be a smooth mapping. We consider the equation $F(u, \lambda)=0$. It defines implicitly a dependence of the state variable $u \in \mathbb{R}^{N}$ on the control parameter $\lambda$.

Let $\Gamma$ be a compact Lie group with a faithful representation in the state space $\mathbb{R}^{N}$ so that we can identify each group element $\gamma \in \Gamma$ with its action on $\mathbb{R}^{N}$, i.e., a linear transformation $\gamma \in \mathbf{G L}(N)$ on $\mathbb{R}^{N}$. We assume the mapping $F$ to be $\Gamma$-equivariant, i.e.,

$$
F(\gamma u, \lambda)=\gamma F(u, \lambda)
$$

for $(u, \lambda) \in \mathbb{R}^{N} \times \mathbb{R}^{1}$ and $\gamma \in \Gamma$. The set Fix $\Gamma \equiv\left\{u \in \mathbb{R}^{N}: \gamma u=u\right.$ for all $\left.\gamma \in \Gamma\right\}$ of fixed points (of $\Gamma$ ) is the set of all states $u \in \mathbb{R}^{N}$ that exhibit the symmetry of the given group $\Gamma$.

Let $\left(u^{*}, \lambda^{*}\right) \in \operatorname{Fix} \Gamma \times \mathbb{R}^{1}$ be a singular point of $F$ with corank $=m \geq 1$, i.e.,

$$
F\left(u^{*}, \lambda^{*}\right)=0, \quad \operatorname{dim} \operatorname{Ker} F_{u}\left(u^{*}, \lambda^{*}\right)=m .
$$

We recall briefly the analysis of the generic spontaneous symmetry breaking bifurcation (see [6, Chapter XIII]):

The solution set of $F(u, \lambda)=0$ is locally identified with the solution set of the bifurcation equation $g(x, t)=0$, where $g: \mathbb{R}^{m} \times \mathbb{R}^{1} \rightarrow \mathbb{R}^{m}, \quad g=g(x, t)$, is the reduced version of $F$ obtained via the classical Liapunov-Schmidt reduction (see [5, Chapter VII]). The space $\mathbb{R}^{m}$ of the reduced state space variables $x$ is identified with $\operatorname{Ker} F_{u}\left(u^{*}, \lambda^{*}\right)$ by the choice of a basis in $\operatorname{Ker} F_{u}\left(u^{*}, \lambda^{*}\right)$. The parameter $t$ is the shift of $\lambda$ namely, $t=\lambda-\lambda^{*}$. The isomorphism between the solution sets of $F$ and $g$ relates the singular point $\left(u^{*}, \lambda^{*}\right) \in \mathbb{R}^{N} \times \mathbb{R}^{1}$ to the origin of $\mathbb{R}^{m} \times \mathbb{R}^{1}$.

The reduced operator $g$ shares the invariance property of $F$ : there exists an $m$-dimensional representation of $\Gamma$ on $\operatorname{Ker} F_{u}\left(u^{*}, \lambda^{*}\right)$ (identified with $\left.\mathbb{R}^{m}\right)$, i.e., a 
homomorphism $\vartheta: \Gamma \rightarrow \mathbf{G L}(m)$, such that $g(\vartheta(\gamma) x, t)=\vartheta(\gamma) g(x, t)$ for each $(x, t) \in \mathbb{R}^{m} \times \mathbb{R}^{1}$ and each group element $\gamma \in \Gamma$. For details, see [5, Proposition 3.3, p. 306].

Generically, $\vartheta$ is an absolutely irreducible representation (see [6, Proposition 3.2, p. 82] ). Apart from the absolute irreducibility, we shall assume $\vartheta$ to be a nontrivial representation; i.e., $\operatorname{Fix}_{\mathbb{R}^{m}} \Gamma \equiv\left\{x \in \mathbb{R}^{m}: \vartheta(\gamma) x=x\right.$ for all $\left.\gamma \in \Gamma\right\}$ is trivial. In other words, $\operatorname{Ker} F_{u}\left(u^{*}, \lambda^{*}\right) \cap \mathrm{Fix} \Gamma=\{0\}$. Consequently, the solution sets $F(u, \lambda)=$ 0 and $g(x, t)=0$, restricted to the symmetric states $u \in \operatorname{Fix} \Gamma$ and $x \in \operatorname{Fix}_{\mathbb{R}^{m}} \Gamma$, respectively, consist (locally) of just one branch that can be parametrised by $\lambda$ and $t$. The Liapunov-Schmidt reduction links the symmetric branch of $F(u, \lambda)=0$ with the trivial branch of $g(x, t)=0$. The other branches, if they exist, do not possess the full symmetry of $\Gamma$.

Let us consider an isotropy subgroup $\Sigma$ of $\Gamma$. We shall assume

$$
\operatorname{dim} \operatorname{Fix}_{\mathbb{R}^{m}} \Sigma=1, \operatorname{Fix}_{\mathbb{R}^{m}} \Sigma \equiv\left\{x \in \mathbb{R}^{m}: \vartheta(\gamma) x=x \text { for all } \gamma \in \Sigma\right\} ;
$$

i.e., $\operatorname{dim}\left(\operatorname{Ker} F_{u}\left(u^{*}, \lambda^{*}\right) \cap \operatorname{Fix} \Sigma\right)=1$. By the Equivariant Branching Lemma (see [6, Theorem 3.3, p. 82]) the operator $g$, restricted as $g:$ Fix $_{\mathbb{R}^{m}} \Sigma \times \mathbb{R}^{1} \rightarrow$ Fix $_{\mathbb{R}^{m}} \Sigma$, generically has a nontrivial solution branch emanating from the origin. By the isomorphism of the solution sets of $F(u, \lambda)=0$ and $g(x, t)=0$, there is a nonsymmetric solution branch of $F:$ Fix $\Sigma \times \mathbb{R}^{1} \rightarrow$ Fix $\Sigma$ emanating from $\left(u^{*}, \lambda^{*}\right)$.

Let us choose a vector $\mathbf{d} \in \mathbb{R}^{m}$ that spans $\operatorname{Fix}_{\mathbb{R}^{m}} \Sigma$; we shall call $\mathbf{d}$ an isotropy vector. It can be identified with a maximal isotropy subgroup, i.e., the largest $\Sigma$ that fixes d. For details, see [6, p. 78]. In what follows, we shall always scale $\mathbf{d}$ as a unit vector, i.e., $\mathbf{d}^{\top} \mathbf{d}=1$.

The symmetry group of the operator $F:$ Fix $\Sigma \times \mathbb{R}^{1} \rightarrow$ Fix $\Sigma$ is the normalizer $\mathcal{N}_{\Gamma}(\Sigma)$ of $\Sigma$ on $\Gamma$, i.e., the largest subgroup of $\Gamma$ that leaves Fix $\Sigma$ invariant. By $[6$, Exercise 2.2, p. 79], the normalizer $\mathcal{N}_{\Gamma}(\Sigma)$ acts on $\operatorname{Fix}_{\mathbb{R}^{m}} \Sigma$ either as the identity or as a $\mathbf{Z}_{2}$-group. Choosing a particular isotropy direction $\mathbf{d}$, we define $h: \mathbb{R}^{1} \times \mathbb{R}^{1} \rightarrow$ $\mathbb{R}^{1}$ by setting

$$
h(\xi, t) \equiv \mathbf{d}^{\top} g(\xi \mathbf{d}, t) .
$$

The solution set of $h(\xi, t)=0$ is constrained to have a trivial branch, namely, $h(0, t) \equiv 0$. If the normalizer acts as the identity, then $h(\cdot, t): \mathbb{R}^{1} \rightarrow \mathbb{R}^{1}$ exhibits no symmetry. If the normalizer acts as $\mathbf{Z}_{2}$, then $h(\cdot, t): \mathbb{R}^{1} \rightarrow \mathbb{R}^{1}$ is $\mathbf{Z}_{2}$-equivariant for each $t$. Hence, $h(-\xi, t)=-h(\xi, t)$ on a neighbourhood of the origin.

Let us use the label $\Gamma$-symmetry breaking bifurcation point for a singular point $\left(u^{*}, \lambda^{*}\right)$ that satisfies the above-resumed assumptions. Such a point is classified by the particular irreducible representation $\vartheta$. Hence, $m$ and $\vartheta$ are group theoretic data that characterise a particular $\Gamma$-symmetry breaking bifurcation point. Moreover, nonsymmetric branches can be referred to (up to a conjugacy) by an isotropy direction $\mathbf{d}$. Obviously, this information matters only if $m \geq 2$. We refer to [6, pp. 132-137], for the discussion of the genericity of the sketched scenario. At any rate, in numerical experiments we shall confine ourselves to $\Gamma$ being a dihedral group $\mathbf{D}_{k}$, where the above-made assumptions are generically satisfied (see [6, Chapter XIII, Section 5]).

Let us consider an $n$-parameter family of vector fields $F: \mathbb{R}^{N} \times \mathbb{R}^{1} \rightarrow \mathbb{R}^{N}$; namely, let

$$
F: \mathbb{R}^{N} \times \mathbb{R}^{1} \times \mathbb{R}^{n} \rightarrow \mathbb{R}^{N}, \quad F=F(u, \lambda, \alpha)
$$


We say that $\left(u^{*}, \lambda^{*}, \alpha^{*}\right) \in \mathbb{R}^{N} \times \mathbb{R}^{1} \times \mathbb{R}^{n}$ is a singular point of (5) if $F\left(\cdot, \cdot, \alpha^{*}\right)$ : $\mathbb{R}^{N} \times \mathbb{R}^{1} \rightarrow \mathbb{R}^{N}$ has a singular point $\left(u^{*}, \lambda^{*}\right)$ in the sense of (2). The classification carries over naturally.

The bifurcation equation at $\left(u^{*}, \lambda^{*}, \alpha^{*}\right)$ obviously depends on the unfolding parameters. In particular, $g(x, t, a)=0$, where

$$
g: \mathbb{R}^{m} \times \mathbb{R}^{1} \times \mathbb{R}^{n} \rightarrow \mathbb{R}^{m}, \quad g=g(x, t, a) .
$$

Note that $\alpha=\alpha^{*}+a$. Again, the reduction links $\left(u^{*}, \lambda^{*}, \alpha^{*}\right)$ with the origin of the domain of $(6)$.

In the $n$-parameter family (5), one may expect degeneracies of the above-sketched scenario of symmetry breaking. For example, the assumption concerning the irreducibility of the representation on $\operatorname{Ker} F_{u}\left(u^{*}, \lambda^{*}, \alpha^{*}\right)$ can be violated. This leads to the scenario of mode interaction. Also, the dimension $m$ of $\operatorname{Ker} F_{u}\left(u^{*}, \lambda^{*}, \alpha^{*}\right)$ might be different from the dimension of $\operatorname{Ker}\left(F_{u}\left(u^{*}, \lambda^{*}, \alpha^{*}\right)\right)^{2}$, as one generically expects for an operator $F: \mathbb{R}^{N} \times \mathbb{R}^{1} \rightarrow \mathbb{R}^{N}$. This leads to a Takens-Bogdanov bifurcation scenario. Let us call all degeneracies affected by the properties of the Jacobian $F_{u}\left(u^{*}, \lambda^{*}, \alpha^{*}\right)$ linear degeneracies. On the other hand, a degeneracy may be caused by nongeneric properties of the Taylor expansion of $F\left(\cdot, \cdot, \alpha^{*}\right): \mathbb{R}^{N} \times \mathbb{R}^{1} \rightarrow \mathbb{R}^{N}$ at $\left(u^{*}, \lambda^{*}\right)$. We refer to [6, pp. 218-223], for an example of a nonlinear degeneracy of a $\mathbf{D}_{3}$-symmetry breaking bifurcation that can happen generically if, in the above agreed terms, $n=1, \Gamma=\mathbf{D}_{3}$, and $m=2$. The representation does not have to be specified, since there is only one 2-dimensional irreducible representation of $\mathbf{D}_{3}$. Let us call this kind of degeneracy a nonlinear degeneracy.

The nonlinear degeneracy of a particular $\Gamma$-symmetry breaking bifurcation point can be classified by the properties of the bifurcation diagram of $g(x, t, 0)=0$, where $x$ is restricted to the one-dimensional state space spanned by a chosen isotropy direction d. Hence now $h$ (see (4)), should be redefined as $h(\xi, t) \equiv \mathbf{d}^{\top} g(\xi \mathbf{d}, t, 0)$. If the normalizer $\mathcal{N}_{\Gamma}(\Sigma)$ acts on $\operatorname{Fix}_{\mathbb{R}^{m}} \Sigma$ as the identity, then, generically, the bifurcation diagram of $h$ consists of the trivial branch and the nontrivial transcritical branch. If the normalizer acts as $\mathbf{Z}_{2}$, then $h$ has generically a (symmetric) pitchfork bifurcation diagram.

\section{Classification of nonlinear Degeneracies}

We have explained our aim to characterise the nonlinear degeneracies via a classification of the scalar bifurcation equation $h(\xi, t)=0$ (see (4)). In this section, we recall the notions of $\mathbf{Z}_{2}$ and $\mathbf{t}$-contact equivalence and present the corresponding classification results in a form that unifies both cases.

Let $\mathcal{E}_{x \lambda}$ be the space of germs of smooth mappings $\mathbb{R}^{2} \rightarrow \mathbb{R}^{1}$ centered at the origin (see [5, p. 55]). Each germ $h \in \mathcal{E}_{x \lambda}$ is represented by a smooth function $h: \mathbb{R}^{2} \rightarrow \mathbb{R}^{1}, h=h(x, \lambda)$, which is defined in a neighbourhood of $(0,0)$. Since this section closely follows [5], we use the standard label for the argument of $h \in \mathcal{E}_{x \lambda}$ in the quoted book.

Let us introduce the following subspaces of $\mathcal{E}_{x \lambda}$ :

$$
\begin{gathered}
\mathcal{E}(\mathbf{t})=\left\{g \in \mathcal{E}_{x \lambda}: g(0, \lambda) \equiv 0\right\}, \\
\mathcal{E}\left(\mathbf{Z}_{2}\right)=\left\{g \in \mathcal{E}_{x \lambda}: g(-x, \lambda)=-g(x, \lambda)\right\} .
\end{gathered}
$$

Remark 3.1. Note that the germ $h$ constructed by means of (4) belongs to either $\mathcal{E}(\mathbf{t})$ or $\mathcal{E}\left(\mathbf{Z}_{2}\right)$ depending on the action of $\mathcal{N}_{\Gamma}(\Sigma)$ on $\operatorname{Fix}_{\mathbb{R}^{m}} \Sigma$. 
Remark 3.2. A germ $g \in \mathcal{E}_{x \lambda}$ belongs to $\mathcal{E}(\mathbf{t})$ and $\mathcal{E}\left(\mathbf{Z}_{2}\right)$ if and only if there exists an $r \in \mathcal{E}_{x \lambda}$ such that $g(x, \lambda)=x r(x, \lambda)$ and $g(x, \lambda)=x r\left(x^{2}, \lambda\right)$, respectively (see [5, p. 129 and Corollary 2.2, p. 249]).

As a consequence, $\mathcal{E}(\mathbf{t})=\mathcal{E}_{u \lambda}\{x\}$ and $\mathcal{E}\left(\mathbf{Z}_{2}\right)=\mathcal{E}_{u \lambda}\{x\}$ with $u=x$ and $u=x^{2}$; i.e., $\mathcal{E}(\mathbf{t})$ and $\mathcal{E}\left(\mathbf{Z}_{2}\right)$ are modules generated by $x$ over the ring $\mathcal{E}_{u \lambda}$ with $u=x$ and $u=x^{2}$.

For the motivation of the following definitions, see [5, Excercise 2.2 on p. 129 and Remark on p. 251].

Definition 3.1. Let $g \in \mathcal{E}(\mathbf{t}), h \in \mathcal{E}(\mathbf{t})$. We say that $g$ is t-contact equivalent with $h$, and write $g \stackrel{\mathbf{t}}{\sim} h$, if there exist $X \in \mathcal{E}_{x \lambda}, \Lambda \in \mathcal{E}_{\lambda}$ and $M \in \mathcal{E}_{x \lambda}$ such that

$$
g(x, \lambda)=M(x, \lambda) h(x X(x, \lambda), \Lambda(\lambda))
$$

and $X(0,0)>0, \Lambda_{\lambda}(0)>0, M(0,0)>0$.

Definition 3.2. Let $g \in \mathcal{E}\left(\mathbf{Z}_{2}\right), h \in \mathcal{E}\left(\mathbf{Z}_{2}\right)$. We say that $g$ is $\mathbf{Z}_{2}$-contact equivalent with $h$, and write $g \stackrel{\mathbf{Z}_{2}}{\sim} h$ if there exist $X \in \mathcal{E}_{x \lambda}, \Lambda \in \mathcal{E}_{\lambda}$ and $M \in \mathcal{E}_{x \lambda}$ such that

$$
g(x, \lambda)=M\left(x^{2}, \lambda\right) h\left(x X\left(x^{2}, \lambda\right), \Lambda(\lambda)\right)
$$

and $X(0,0)>0, \Lambda_{\lambda}(0)>0, M(0,0)>0$.

The codimensions $\operatorname{codim}_{\mathbf{t}}(g)$ and $\operatorname{codim}_{\mathbf{Z}_{2}}(g)$ of germs $g \in \mathcal{E}(\mathbf{t})$ and $g \in \mathcal{E}\left(\mathbf{Z}_{2}\right)$ under $\mathbf{t}$ and $\mathbf{Z}_{2}$-equivalence are defined in [5, Excersise 2.3, p. 129 and Definition 3.1 on p. 258], respectively.

Remark 3.3. If $r \in \mathcal{E}_{u \lambda}$, then $\operatorname{codim}_{\mathbf{t}}(r)$ is defined as the codimension of $T(r ; \mathbf{t}) \equiv$ $\left\langle r, u r_{u}\right\rangle+\mathcal{E}_{\lambda}\left\{r_{\lambda}\right\}$ in $\mathcal{E}_{u \lambda} ; u$ is just a label for a $1-\mathrm{D}$ state variable. The subspace $T(r ; \mathbf{t})$ could be interpreted as the formal tangent space to $r=r(u, \lambda)$ under $\mathbf{t}$ equivalence on $\mathcal{E}_{u \lambda}$. Note that if $g(x, \lambda)=x r(u, \lambda), u=x$ and $u=x^{2}$, respectively, then the tangent spaces $T(g ; \mathbf{t})$ and $T\left(g ; \mathbf{Z}_{2}\right)$ to $g$ under $\mathbf{t}$ and $\mathbf{Z}_{2}$-equivalence factor through $x$, see [5, formulae (2.22) and (3.2) on pp. 129 and 258]. In fact, $T(g ; \mathbf{t})=T(r ; \mathbf{t})\{x\}, u=x$, and $T\left(g ; \mathbf{Z}_{2}\right)=T(r ; \mathbf{t})\{x\}, u=x^{2}$. By using the factorisations $\mathcal{E}(\mathbf{t})=\mathcal{E}_{u \lambda}\{x\}$ and $\mathcal{E}\left(\mathbf{Z}_{2}\right)=\mathcal{E}_{u \lambda}\{x\}$ with $u=x$ and $u=x^{2}$ one can prove that $\operatorname{codim}_{\mathbf{t}}(r)=\operatorname{codim}_{\mathbf{t}}(g)$, and $\operatorname{codim}_{\mathbf{t}}(r)=\operatorname{codim}_{\mathbf{Z}_{2}}(g)$, respectively.

In [5, pp. 263-267], all $\mathbf{Z}_{2}$-equivalence classes of germs $g \in \mathcal{E}\left(\mathbf{Z}_{2}\right)$ with $\operatorname{codim}_{\mathbf{Z}_{2}}(g)$ $\leq 3$ are described. Though there is not a direct reference, it is clear that an analogous description holds for t t-equivalence classes on $\mathcal{E}(\mathbf{t})$ with $\operatorname{codim}_{\mathbf{t}}(g) \leq 3$. For some brief comments on this theme, see the following

Remark 3.4. If $g \in \mathcal{E}\left(\mathbf{Z}_{2}\right)$ and $g(x, \lambda)=x r(u, \lambda), u=x^{2}$, then the orbit of $g$ under $\mathbf{Z}_{2}$-equivalence is naturally linked with the orbit of $r \in \mathcal{E}_{u \lambda}$ under tequivalence. In particular, $g \stackrel{\mathbf{Z}_{2}}{\sim} h, h(x, \lambda)=x s(u, \lambda), u=x^{2}, \operatorname{codim}_{\mathbf{Z}_{2}}(g)<\infty$, if and only if $r \stackrel{\mathbf{t}}{\sim} s, \operatorname{codim}_{\mathbf{t}}(r)<\infty$. Similarly, if $g \in \mathcal{E}(\mathbf{t}), g(x, \lambda)=x r(x, \lambda)$, and $h \in \mathcal{E}(\mathbf{t}), h(x, \lambda)=x s(x, \lambda)$, then $g \stackrel{\mathbf{t}}{\sim} h($ in $\mathcal{E}(\mathbf{t}))$ if and only if $r \stackrel{\mathbf{t}}{\sim} s$ (in $\left.\mathcal{E}_{x \lambda}\right)$. Hence, [5, Tables 5.2-5.4 on pp. 264-266], classify t-equivalence classes on $\mathcal{E}_{u \lambda}$ with codimension $\leq 3$.

Theorem 3.1. Let $g(x, \lambda)=x r(u, \lambda), u=x$, be a germ in $\mathcal{E}(\mathbf{t})$ with $\operatorname{codim}_{\mathbf{t}}(g) \leq$ 3 , and let $g(x, \lambda)=x r(u, \lambda), u=x^{2}$, be a germ in $\mathcal{E}\left(\mathbf{Z}_{2}\right)$ with $\operatorname{codim}_{\mathbf{Z}_{2}}(g) \leq \overline{3}$, respectively. Let $r(0,0)=0$. Then the germ $r \in \mathcal{E}_{u \lambda}$ is $\mathbf{t}$-equivalent to one of the normal forms listed in Table 1. 
TABLE 1. Normal forms of t-contact equivalence classes on $\mathcal{E}_{u \lambda}$

\begin{tabular}{|c|c|c|}
\hline $\begin{array}{l}\text { type } \\
\text { /codim }\end{array}$ & $\begin{array}{l}\text { Normal Form } \\
\epsilon= \pm 1, \delta= \pm 1, \phi= \pm 1\end{array}$ & $\begin{array}{l}\text { Defining equations } \\
\text { / Nondegeneracy conditions }\end{array}$ \\
\hline $\begin{array}{l}1 \\
1\end{array}$ & $\begin{array}{l}\epsilon u+\delta \lambda \\
\epsilon=\operatorname{sgn}\left(r_{u}\right) ; \delta=\operatorname{sgn}\left(r_{\lambda}\right)\end{array}$ & $\begin{array}{l}r=0 \\
r_{\lambda} \neq 0\end{array}$ \\
\hline $\begin{array}{l}2 \mathrm{a}, 2 \mathrm{~b}, 2 \mathrm{c} \\
/ k=1,2,3\end{array}$ & $\begin{array}{l}\epsilon u^{k+1}+\delta \lambda, 1 \leq k \leq 3 \\
\epsilon=\operatorname{sgn}\left(r_{u^{k+1}}\right) ; \delta=\operatorname{sgn}\left(r_{\lambda}\right)\end{array}$ & $\begin{array}{l}r=\cdots=r_{u^{k}}=0 \\
r_{u^{k+1}} \neq 0 ; r_{\lambda} \neq 0\end{array}$ \\
\hline $\begin{array}{l}3 \mathrm{a}, 3 \mathrm{~b}, 3 \mathrm{c} \\
/ k=1,2,3\end{array}$ & $\begin{array}{l}\epsilon u+\delta \lambda^{k+1} \\
\epsilon=\operatorname{sgn}\left(r_{u}\right) ; \delta=\operatorname{sgn}\left(r_{\lambda^{k+1}}\right)\end{array}$ & $\begin{array}{l}r=\cdots=r_{\lambda^{k}}=0 \\
r_{u} \neq 0 ; r_{\lambda^{k+1}} \neq 0\end{array}$ \\
\hline $\begin{array}{l}4 \\
\end{array}$ & $\begin{array}{l}\epsilon u^{2}+2 m \lambda u+\delta \lambda^{2} \\
\epsilon=\operatorname{sgn}\left(r_{u u}\right) ; \delta=\operatorname{sgn}\left(r_{\lambda \lambda}\right) ; \\
m^{2} \neq \epsilon \delta, m=r_{u \lambda} / \sqrt{\left|r_{u u} r_{\lambda \lambda}\right|}\end{array}$ & $\begin{array}{l}r=r_{u}=r_{\lambda}=0 \\
r_{u u} \neq 0 ; r_{\lambda \lambda} \neq 0 ; D_{2}(r) \neq 0\end{array}$ \\
\hline $\begin{array}{l}5 \\
/ 3\end{array}$ & $\begin{array}{l}\epsilon u^{2}+2 \delta \lambda u+\phi \lambda^{3} \\
\epsilon=\operatorname{sgn}\left(r_{u u}\right) ; \delta=\operatorname{sgn}\left(r_{u \lambda}\right) \\
\phi=\operatorname{sgn}\left(r_{\lambda \lambda \lambda}\right)\end{array}$ & $\begin{array}{l}r=r_{u}=r_{\lambda}=r_{\lambda \lambda}=0 \\
r_{u u} \neq 0 ; r_{\lambda \lambda \lambda} \neq 0 ; D_{2}(r) \neq 0\end{array}$ \\
\hline $\begin{array}{l}6 \\
/ 3\end{array}$ & $\begin{array}{l}\epsilon u^{3}+\delta \lambda u+\phi \lambda^{2} \\
\epsilon=\operatorname{sgn}\left(r_{u u u}\right) ; \delta=\operatorname{sgn}\left(r_{u \lambda}\right) \\
\phi=\operatorname{sgn}\left(r_{\lambda \lambda}\right)\end{array}$ & $\begin{array}{l}r=r_{u}=r_{u u}=r_{\lambda}=0 \\
r_{\text {uuu }} \neq 0 ; r_{\lambda \lambda} \neq 0 ; D_{2}(r) \neq 0\end{array}$ \\
\hline $\begin{array}{l}7 \\
/ 3\end{array}$ & $\begin{array}{l}\phi u^{3}+\epsilon u^{2}+2 \delta \lambda u+\epsilon \lambda^{2} \\
\epsilon=\operatorname{sgn}\left(r_{u u}\right) ; \delta=\operatorname{sgn}\left(r_{u \lambda}\right) \\
\phi=-\operatorname{sgn}\left(r_{u \lambda} r_{u u} D_{3}(r)\right)\end{array}$ & $\begin{array}{l}r=r_{u}=r_{\lambda}=D_{2}(r)=0 \\
r_{u u} \neq 0 ; r_{\lambda \lambda} \neq 0 ; D_{3}(r) \neq 0\end{array}$ \\
\hline
\end{tabular}

Proof. The proof consists in the interpretation of the result in [5, Theorem 5.1 on p. 263].

Remark 3.5. It is understood that the singularity type that Theorem 3.1 assigns to $r$ via Table 1, is also assigned to the corresponding $g$. The normal form of $r$, say $s \in \mathcal{E}_{u \lambda}$, yields a normal form $h \in \mathcal{E}_{x \lambda}$ of $g$; namely, $h(x, \lambda)=x s(u, \lambda)$, where $u=x$ and $u=x^{2}$, respectively. The value of codim in Table 1 is to be interpreted as $\operatorname{codim}_{\mathbf{t}}(g)$ or $\operatorname{codim}_{\mathbf{Z}_{2}}(g)$, as appropriate.

In Table 1 , the values of the parameters $\epsilon, \delta$ and $\phi$ are defined by means of derivatives of $r$ at the origin. From the numerical point of view, the algebraicgeometric classification is the most important: $g(x, \lambda)=x r(u, \lambda)$ is of a type listed in Table 1 if and only if the factor $r \in \mathcal{E}_{u \lambda}$ satisfies the relevant defining equations and nondegeneracy conditions.

Remark 3.6. The parameter $m$ in the normal form of the singularity type is a modal parameter (see [5, Remark 5.2 (ii) on p. 265]). Hence, it is simultaneously an unfolding parameter. We shall consider the singularity type 4 as a moduli family (see $[5,(6.3)$ on p. 239]). The topological codimension of this family is 2 .

\section{NUMERICAL DETECTION}

The standard numerical tool for detection and pathfollowing of steady state bifurcation points (without symmetry) is the generalised Liapunov-Schmidt reduction (see [9]). For recent developments of the reduction technique that make use 
of bordered matrices to define projectors, see [7]. Finally, the Liapunov-Schmidt reduction can be applied to operator equations in Banach spaces (see [2]).

The equivariant reduction procedure is proposed in [8]. The approach is related to the construction of test functions for symmetry breaking bifurcation by equivariant bordered Jacobians (see e.g., [10], [3]). Let us recall the idea of the reduction.

We consider the mapping $F$ (see (5)). Given $m$, we consider a pair of full rank matrices $M \in \mathcal{L}\left(\mathbb{R}^{m}, \mathbb{R}^{N}\right), L \in \mathcal{L}\left(\mathbb{R}^{N}, \mathbb{R}^{m}\right)$. We define the (open) set $\mathfrak{D} \equiv$ $\{(u, \lambda, \alpha): \operatorname{det} \mathcal{J}(u, \lambda, \alpha) \neq 0\}$, where

$$
\mathcal{J}(u, \lambda, \alpha)=\left(\begin{array}{cc}
F_{u}(u, \lambda, \alpha) & M \\
L & O
\end{array}\right) \in \mathcal{L}\left(\mathbb{R}^{N+m}, \mathbb{R}^{N+m}\right) ;
$$

$O \in \mathcal{L}\left(\mathbb{R}^{m}, \mathbb{R}^{m}\right)$ is a null matrix.

Let $(u, \lambda, \alpha) \in \mathfrak{D}$ be fixed. Given $(x, t, a) \in \mathbb{R}^{m} \times \mathbb{R}^{1} \times \mathbb{R}^{n}$, we define implicitly $g \in \mathbb{R}^{m}$ and $v \in \mathbb{R}^{N}$ via the following system of $N+m$ nonlinear equations:

$$
F(u+v, \lambda+t, \alpha+a)-M g=F(u, \lambda, \alpha), \quad L v=x .
$$

Definition 4.1. Let $\vartheta: \Gamma \rightarrow \mathbf{G L}(m)$ be a particular $m$-dimensional representation of $\Gamma$. If a pair of full rank matrices $M \in \mathcal{L}\left(\mathbb{R}^{m}, \mathbb{R}^{N}\right), L \in \mathcal{L}\left(\mathbb{R}^{N}, \mathbb{R}^{m}\right)$ satisfy

$$
\vartheta(\gamma) L=L \gamma, \quad M \vartheta(\gamma)=\gamma M
$$

for each $\gamma \in \Gamma$, we say that $M, L$ are symmetry adapted bordering matrices (with respect to $\Gamma$ and $\vartheta$ ).

For the construction of symmetry adapted bordering matrices, see e.g., [8], [10]. Let us resume the basic idea for finite groups $\Gamma$ : Given a unit vector $\mathbf{e} \in \mathbb{R}^{m}$ and a seed vector $\xi \in \mathbb{R}^{N}, \xi \neq 0$, we can set $M=\sum_{\gamma \in \Gamma} \gamma \xi \mathbf{e}^{\top} \vartheta\left(\gamma^{-1}\right) \in \mathcal{L}\left(\mathbb{R}^{m}, \mathbb{R}^{N}\right)$. A similar formula defines $L^{\top}$; we may choose a different $\mathbf{e}$ and $\xi$. It is easy to check that $M$ and $L$ satisfy (10).

Observation 4.1. Let $M$ and $L$ be symmetry adapted bordering matrices with respect to $\Gamma$ and a representation $\vartheta: \Gamma \rightarrow \mathbf{G L}(m)$. Let us consider the relevant $\mathfrak{D}$ and choose a point $(u, \lambda, \alpha) \in \mathfrak{D}$. Then

$$
\vartheta(\gamma) g(x, t, a ; u, \lambda, \alpha)=g(\vartheta(\gamma) x, t, a ; \gamma u, \lambda, \alpha)
$$

for each $\gamma \in \Gamma$ and each $(x, t, a)$ from the domain of both $g(\cdot, \cdot, \cdot ; u, \lambda, \alpha)$.

Proof. See [8, Theorem 2.2].

Let $\vartheta: \Gamma \rightarrow \mathbf{G L}(m)$ be an $m$-dimensional irreducible representation of $\Gamma$. Let $\mathbf{d} \in \mathbb{R}^{m}$ be an isotropy direction; namely, let $\operatorname{span}\{\mathbf{d}\}=\operatorname{Fix}_{\mathbb{R}^{m}} \Sigma$ and $\mathbf{d}^{\top} \mathbf{d}=1$. If $(u, \lambda, \alpha) \in \operatorname{Fix} \Gamma \times \mathbb{R}^{1} \times \mathbb{R}^{n}$, then as a consequence of Observation 4.1

$$
g(\cdot, \cdot, \cdot ; u, \lambda, \alpha): \operatorname{Fix}_{\mathbb{R}^{m}} \Sigma \times \mathbb{R}^{1} \times \mathbb{R}^{n} \rightarrow \operatorname{Fix}_{\mathbb{R}^{m}} \Sigma .
$$

Hence, we may define $h(\cdot, \cdot, \cdot ; u, \lambda, \alpha) \in \mathcal{E}_{1+1+n}$ as

$$
h(x, t, a ; u, \lambda, \alpha)=\mathbf{d}^{\top} g(x \mathbf{d}, t, a ; u, \lambda, \alpha)
$$

for each $(x, t, a)$ from a small neighbourhood of the origin $0 \in \mathbb{R}^{1} \times \mathbb{R}^{1} \times \mathbb{R}^{n}$.

The singular points of a given type are sought as regular roots of

$$
\mathcal{F}: \text { Fix } \Gamma \times \mathbb{R}^{1} \times \mathbb{R}^{n} \rightarrow \text { Fix } \Gamma \times \mathbb{R}^{\operatorname{codim}+1}, \quad n \geq \operatorname{codim},
$$


where $\mathcal{F}(u, \lambda, \alpha) \equiv\left\{F(u, \lambda, \alpha) ; f_{1}(u, \lambda, \alpha), \ldots, f_{\text {codim }+1}(u, \lambda, \alpha)\right\}$ while $f_{i}(u, \lambda, \alpha)$ : Fix $\Gamma \times \mathbb{R}^{1} \times \mathbb{R}^{n} \rightarrow \mathbb{R}^{1}$ are the particular test functions. The test functions can be retrieved from Table 1 (defining equations); see [1] for details.

\section{Numerical EXAmple}

We developed a code in MATLAB 4.0 for the detection and pathfollowing of $\Gamma$-symmetry breaking bifurcation points discussed in this paper. The aim was to verify that the proposed numerical treatment can really work. By the restrictions of the programming environment we could treat small dimensional problems only.

In the following example we consider the 4-box Brusselator (see [3]). It yields a mapping (5) with $N=8$ and $n=2$; we denote the parameter vector $(B, A)$ by $\alpha$. The number of parameters suggests that singularities with (topological) codim $\leq 2$ (see Table 1) can be expected and indeed they were found. The problem has the symmetry of the group $\Gamma=\mathbf{D}_{4}=\left\{\iota, \kappa, \rho, \kappa \rho, \kappa \rho^{2}, \kappa \rho^{3}, \rho^{2}, \rho^{3}\right\}$; the actions of the flip $\kappa$ and the rotation $\rho$ are described in [3]. The particular action admits the following nontrivial irreducible representations:

$$
\vartheta^{(2)}(\kappa)=\left(\begin{array}{cc}
1 & 0 \\
0 & -1
\end{array}\right), \quad \vartheta^{(2)}(\rho)=\left(\begin{array}{cc}
0 & -1 \\
1 & 0
\end{array}\right), \quad \vartheta^{(1)}(\kappa)=1, \vartheta^{(1)}(\rho)=-1 .
$$

We have used $L^{(2)}=\frac{1}{2}\left(\begin{array}{cccccccc}1 & 0 & 0 & 0 & -1 & 0 & 0 & 0 \\ 0 & 0 & 1 & 0 & 0 & 0 & -1 & 0\end{array}\right), M^{(2)}=L^{(2)^{\top}}$, and $L^{(1)}=\frac{1}{4}\left(\begin{array}{cccccccc}1 & 0 & -1 & 0 & 1 & 0 & -1 & 0\end{array}\right), M^{(1)}=L^{(1)^{\top}}$, as the relevant symmetry adapted bordering matrices in the tests reported below.

In the case of the representation $\vartheta^{(2)}$, there are two nonconjugate isotropy directions, namely, $\mathbf{d}^{(2)}=(1,0)^{\top}$ and $\mathbf{d}^{(2)}=\frac{\sqrt{2}}{2}(1,-1)^{\top}$, plus one conjugate copy of each. The relevant isotropy subgroups are $\Sigma^{(2)}=\{\iota, \kappa\}$ and $\Sigma^{(2)}=\{\iota, \kappa \rho\}$, respectively. Both $\Sigma^{(2)}$ are representations of $\mathbf{Z}_{2}$.

The 1-dimensional representation $\vartheta^{(1)}$ has $\mathbf{d}^{(1)}=1$ as an isotropy direction. The relevant (maximal) isotropy subgroup is $\Sigma^{(1)}=\left\{\iota, \kappa, \kappa \rho^{2}, \rho^{2}\right\}$. Hence, it is a representation of $\mathbf{Z}_{2} \oplus \mathbf{Z}_{2}$.

The normalizer $\mathcal{N}_{\Gamma}(\Sigma)$ acts on each of the spaces $\operatorname{span}\left\{\mathbf{d}^{(j)}\right\}, j=1,2$, as $\mathbf{Z}_{2}$. Hence, the classification of $\mathbf{Z}_{2}$-equivalence classes in Section 3 will be appropriate for our characterisation of nonlinear degeneracies.

In Figure 1, we show (in the projection onto the unfolding parameter space) all detected $\mathbf{D}_{4}$-symmetry breaking bifurcation points with codim $\geq 1$. There is always a pair of symmetry breaking bifurcation points of the same type that correspond to each of the depicted unfolding parameters. They have different $\lambda$ and state variable values and also differ by the group theoretic data (namely, by the representations $\vartheta^{(2)}$ and $\left.\vartheta^{(1)}\right)$. It should be noted that the degeneracy $r_{u}=0$ that defines the type 2a-points is, in the case of $\vartheta^{(2)}$, detected only along the isotropy direction $\mathbf{d}^{(2)}=\frac{\sqrt{2}}{2}(1,-1)^{\top}$ and its conjugate.

The imperfect bifurcation analysis in a neighbourhood of the detected type 4 -singular point is significantly affected by the mode interaction (namely, the $\vartheta^{(2)}$ mode colliding with the $\vartheta^{(1)}$-mode). This is also a phenomenon with codim $=1$. In the scale of Figure 1, the projection of these mode interaction points onto the unfolding parameter space is a curve very close to the curve of type 2a-singular points. The zoom on Figure 1 can distinguish the mode interaction branch. 

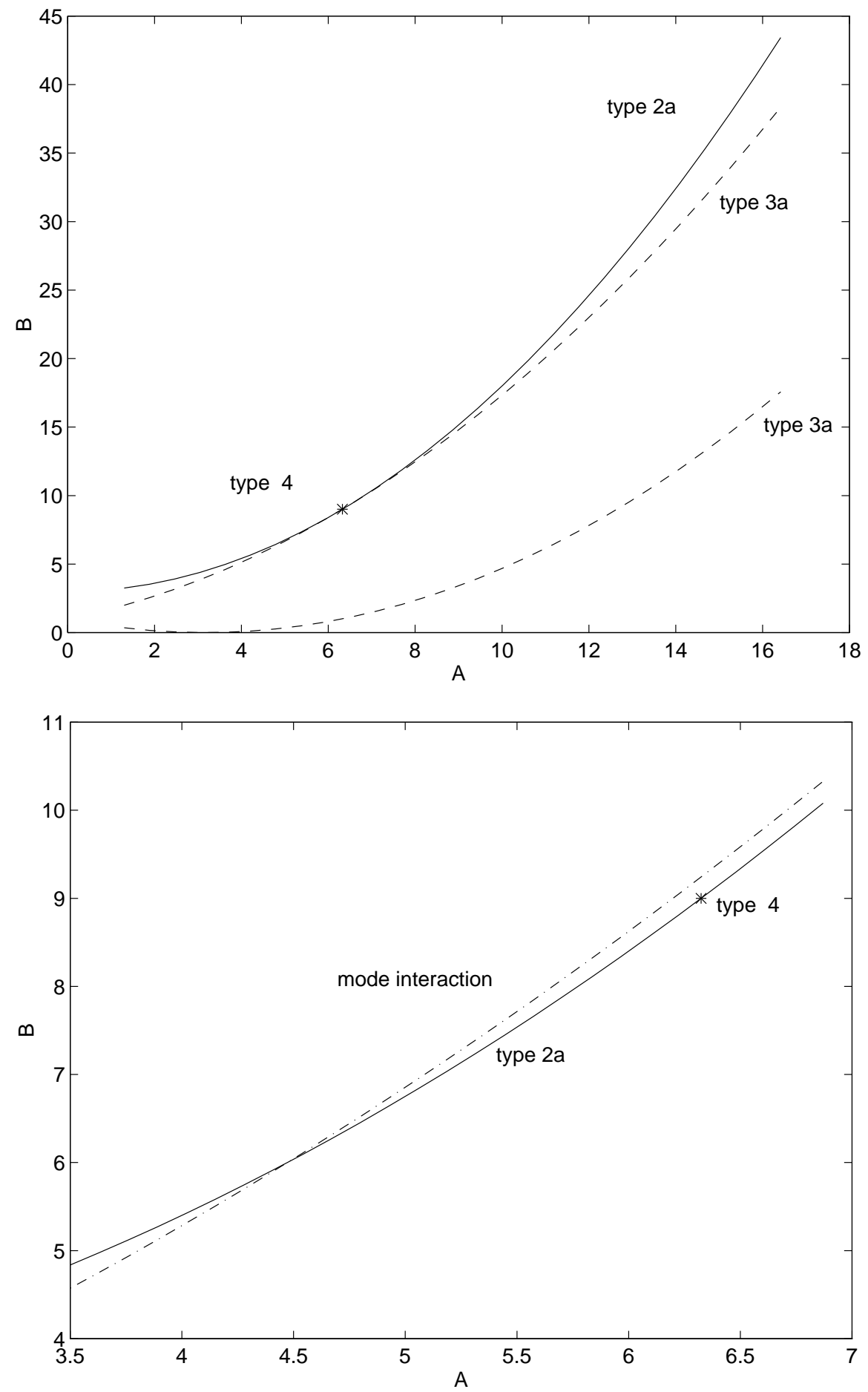

Figure 1. 4-box Brusselator: Symmetry breaking bifurcation points with codim $=1$ and codim $=2$. On the bottom: Zoom-in with the branch of mode interaction points. 
1106 KLAUS BÖHMER, WILLY GOVAERTS, AND VLADIMÍR JANOVSKÝ
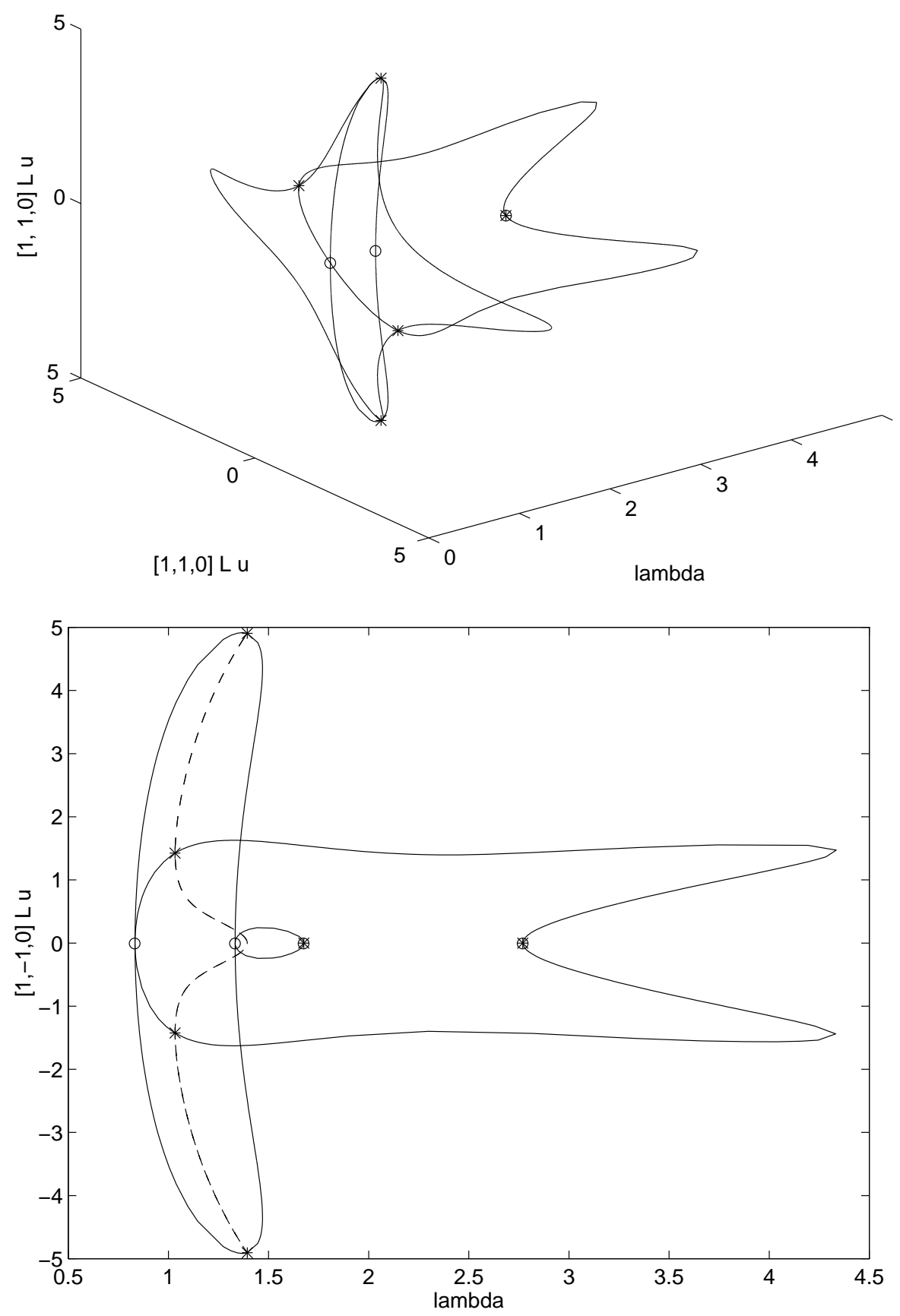

FiguRE 2. 4-box Brusselator: Secondary and tertiary branches. 


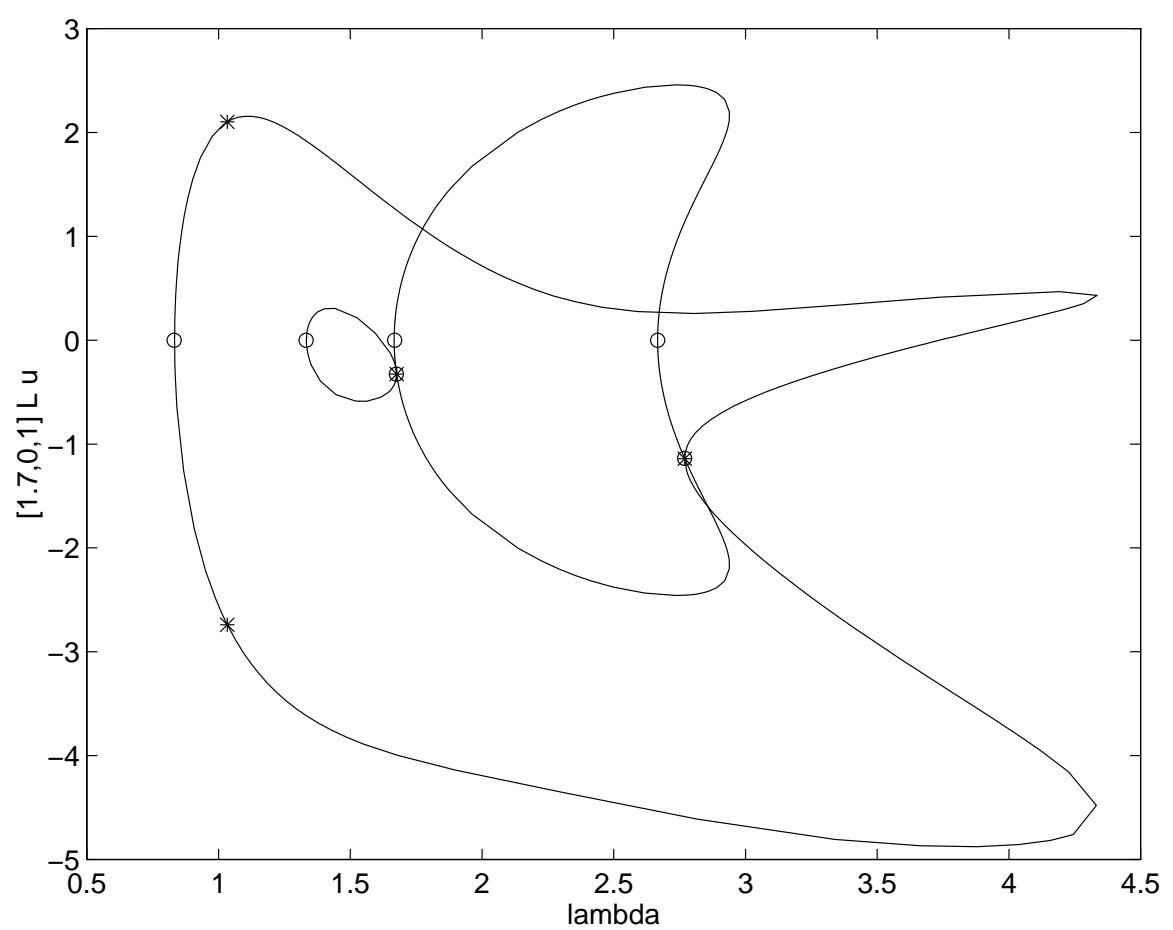

FiguRE 3. 4-box Brusselator: Secondary branches.

Since two modes are involved in the $\Gamma$-symmetry breaking numerical analysis, it is natural to define the 3 -D reduced vector field $g(\cdot, \cdot, \cdot ; u, \lambda, \alpha): \mathbb{R}^{3} \times \mathbb{R}^{1} \times$ $\mathbb{R}^{2} \rightarrow \mathbb{R}^{3}$ by means of the bordering matrices $M=\left(M^{(2)}, M^{(1)}\right) \in \mathcal{L}\left(\mathbb{R}^{3}, \mathbb{R}^{8}\right)$, $L=M^{\top} \in \mathcal{L}\left(\mathbb{R}^{8}, \mathbb{R}^{3}\right)$. Obviously, $M$ and $L$ are symmetry adapted w.r.t. $\Gamma$ and the representation $\vartheta=\operatorname{diag}\left(\vartheta^{(2)}, \vartheta^{(1)}\right)$. The isotropy directions are $\mathbf{d}=(1,0,0)^{\top}$, $\mathbf{d}=\frac{\sqrt{2}}{2}(1,-1,0)^{\top}, \mathbf{d}=(0,0,1)^{\top}$, and their conjugates. For each of these isotropy directions, one can formally proceed with the definitions of the test functions for nonlinear degeneracies. Simultaneously, the particular definition of $g$ allows us to construct test functions for the mode interaction (see e.g., [8]).

To illustrate the complexity of an unfolding of the type 4-singularity in the example studied, let us consider $\alpha=(8.5,6)^{\top}$. Significant branches of the bifurcation diagram $\left\{(u, \lambda) \in \mathbb{R}^{8} \times \mathbb{R}^{1}: F(u, \lambda, \alpha)=0\right\}$ are depicted in Figure 2. The circles mark the $\Gamma$-symmetry breaking bifurcation points of type 1 . These are the primary bifurcation points. The secondary branches that emanate from these points have $\Sigma$ symmetry. In Figure 2, they have $\Sigma^{(2)}$-symmetry. The kidney-like branch in Figure 3 has $\Sigma^{(1)}$-symmetry, while the remaining two branches have $\Sigma^{(2)}$-symmetry.

The secondary bifurcation points organized by the type 4-bifurcation point (corresponding to the $\vartheta^{(2)}$ representation) are marked as stars. The star-shaped tertiary branch in Figure 3 makes a closed loop connecting both visualised secondary branches and their conjugate copies.

The remaining secondary bifurcation points (marked as the combined circles and stars) are inherited from the above mentioned mode interaction. In these bifurcation points, the $\mathbf{Z}_{2} \oplus \mathbf{Z}_{2}$-symmetry of the kidney-like secondary branch in 
Figure 3 is broken into $\mathbf{Z}_{2}$-symmetry of the secondary branches emanating from both type 1-primary bifurcation points (with $\vartheta^{(2)}$ representation) in the isotropy direction $\mathbf{d}=(1,0,0)^{\top}$ (and its conjugate, respectively).

\section{REFERENCES}

[1] K. Böhmer, W. Govaerts And V. Janovský, Numerical detection of symmetry breaking bifurcation points with nonlinear degeneracies, Bericht zur Fachbereich Mathematik der Philipps-Universität Marburg 1996.

[2] K. BöHmer On a numerical Lyapunov-Schmidt method for operator equations, Computing 51, pp. 237-269, 1993. MR 95a:65100

[3] M. Dellnitz and B. Werner, Computational methods for bifurcation problems with symmetries - with special attention to steady state and Hopf bifurcation points, J. Comput. Appl. Math., 26:97-123, 1989. MR 91a:58133

[4] K. Gatermann and R. Lauterbach, Automatic classification of normal forms, Preprint SC 95-3 (Februar 1995), Konrad-Zuse-Zentrum für Informationstechnik Berlin, Germany

[5] M. Golubitsky and D. Schaeffer, Singularities and Groups in Bifurcation Theory, Vol. 1, Springer Verlag, New York, 1985. MR 86e:58014

[6] M. Golubitsky, I. Stewart And D. Schaeffer, Singularities and Groups in Bifurcation Theory, Vol. 2, Springer Verlag, New York, 1988. MR 89m:58038

[7] W. Govaerts, Computation of singularities in large nonlinear systems, SIAM J. Num. Anal. 34 (1997) pp. 867-880. CMP 97:13

[8] V. JanovskÝ And P. Plecháč, Numerical applications of equivariant reduction techniques, in Bifurcation and Symmetry, M. Golubitsky. E. Allgower, K. Böhmer, ed., Birkhäser Verlag, 1992. CMP 94:04

[9] A. D. JEPSON AND A. Spence, On a reduction process for nonlinear equations, SIAM J. Math. Anal., 20 (1989), pp. 39-56. MR 90b:58018

[10] B. Werner, The numerical analysis of bifurcation problems with symmetries based on bordered Jacobians, In Exploiting Symmetry in Applied and Numerical Analysis, E. L. Allgower, K. Georg, and R. Miranda, editors, Volume 29 of Lectures in Applied Mathematics, pages 443-457, American Mathematical Society, Providence, RI, 1993. MR 94k:65075

Philipps Universität, Fachbereich Mathematik, Marburg, Germany

E-mail address: boehmer@mathematik. uni-marburg.de

Department of Applied Mathematics and Computer Science, University of Genh, BELGIUM

E-mail address: Willy.Govaerts@rug.ac.be

Faculty of Mathematics and Physics, Charles University, Prague, Czech Republik

E-mail address: janovsky@ms.mff.cuni.cz 